\title{
The impact of dermoscopy on melanoma detection in the practice of dermatologists in Europe: results of a pan-European survey
}

\author{
A.M. Forsea, ${ }^{1, *}$ P. Tschandl, ${ }^{2}$ I. Zalaudek, ${ }^{3}$ V. del Marmol, ${ }^{4}$ H.P. Soyer, ${ }^{5}$ Eurodermoscopy Working Group ${ }^{\dagger}$ \\ G. Argenziano, ${ }^{6}$ A.C. Geller ${ }^{7}$ \\ ${ }^{1}$ Dermatology Department, Elias University Hospital, Carol Davila University of Medicine and Pharmacy, Bucharest, Romania \\ ${ }^{2}$ Department of Dermatology, Medical University of Vienna, Vienna, Austria \\ ${ }^{3}$ Non-Melanoma Skin Cancer Unit, Department of Dermatology and Venereology, Medical University of Graz, Graz, Austria \\ ${ }^{4}$ Dermatology Department, Hopital Erasme, Universite Libre de Bruxelles, Bruxelles, Belgium \\ ${ }^{5}$ Dermatology Research Centre, School of Medicine, Translational Research Institute, The University of Queensland, Brisbane, Qld, Australia \\ ${ }^{6}$ Dermatology Unit, Second University of Naples, Naples, Italy \\ ${ }^{7}$ Social and Behavioral Sciences, Harvard T.H. Chan School of Public Health, Harvard University, Boston, MA, USA \\ ${ }^{*}$ Correspondence: A.M. Forsea. E-mail: aforsea@yahoo.com
}

\begin{abstract}
Background Dermoscopy is a widely used technique that can increase the sensitivity and specificity of melanoma detection. Information is lacking on the impact of dermoscopy use on the detection of melanoma in the real-life practice of European dermatologists.

Objective To identify factors that influence the benefit of using dermoscopy for increasing melanoma detection and lowering the number of unnecessary biopsies in the practice of European dermatologists.

Methods We conducted a survey of dermatologists registered in 32 European countries regarding the following: the demographic and practice characteristics, dermoscopy training and use, opinions on dermoscopy and the self-estimated impact of dermoscopy use on the number of melanomas detected and the number of unnecessary biopsies performed in practice.

Results Valid answers were collected for 7480 respondents, of which 6602 reported using dermoscopy. Eighty-six per cent of dermoscopy users reported that dermoscopy increased the numbers of melanomas they detected, and $70 \%$ reported that dermoscopy decreased the number of unnecessary biopsies of benign lesions they performed. The dermatologists reporting these benefits were more likely to have received dermoscopy training during residency, to use dermoscopy frequently and intensively, and to use digital dermoscopy systems and pattern analysis compared to dermatologists who did not perceive any benefit of dermoscopy for the melanoma recognition in their practice.

Conclusions Improving dermoscopy training, especially during residency and increasing access to digital dermoscopy equipment are important paths to enhance the benefit of dermoscopy for melanoma detection in the practice of European dermatologists.
\end{abstract}

Received: 6 October 2016; Accepted: 2 January 2017

\section{Conflicts of interest}

None declared.

Funding source

None declared.

\section{Introduction}

Dermoscopy is an established tool for the clinical diagnosis of a wide range of skin diseases, ${ }^{1-8}$ but its main role remains the facilitation of melanoma diagnosis. Dermoscopy used by trained

${ }^{\dagger}$ Eurodermoscopy Working Group members details are in Appendix 1. physicians has been shown to increase the sensitivity of melanoma detection up to ninefold compared to the clinical examination alone. ${ }^{9-11}$ Moreover, it allows for the earlier detection of thinner melanomas ${ }^{12,13}$ by revealing subtle changes of melanocytic lesions, invisible to the naked eye. As such, it is a valuable aid for melanoma screening and for the monitoring of high-risk 
patients, which is recommended by the current practice guidelines for melanoma diagnosis. ${ }^{14-17}$ At the same time, multiple studies have shown that dermoscopy use at an expert level can increase the specificity of melanoma diagnosis, which translates into the reduction in unnecessary biopsies of benign lesions. ${ }^{18-20}$ These results are supported by indirect evidence from the analysis of dermatological practices, ${ }^{20-22}$ where the number needed to excise (NNE), calculated as the number of all melanocytic lesions excised for each confirmed melanoma, reportedly decreases from 20 to 40 in general dermatology practices to $4-8$ in specialized skin cancer clinics using dermoscopy.

The proven capacity of dermoscopy to increase the diagnostic accuracy for melanoma suggests that this technique, if used systematically, could have a population-wide impact, by improving early detection and hence the prognosis of melanoma, while reducing the need for invasive diagnostic procedures and their related human and material costs. So far, most evidence on the impact of dermoscopy on melanoma early detection comes from controlled studies in several expert centres, involving a limited number of dermatologists. However, very little is known on how dermoscopy actually impacts everyday dermatology practice in Europe ${ }^{18,23}$ and on the factors that enhance or limit the benefits that dermatologists derive from its use regarding melanoma diagnosis.

In this context, we conducted the first pan-European survey of the patterns, motivations and obstacles of dermoscopy use. Herein, we explored the perceptions of European dermatologists about the benefits of dermoscopy in increasing their capacity to detect melanomas and reducing the number of unnecessary biopsies. We analysed further the demographic, practice- and training-related factors that influence these perceptions.

\section{Methods}

The Eurodermoscopy pan-European survey of dermatologists was conducted under the auspices of the International Dermoscopy Society (IDS), and its methodology and detailed data handling were described in detail elsewhere. ${ }^{24}$ In brief, the study instrument consisted of a 20 -item questionnaire, ${ }^{24}$ covering demographic, practice-related and dermoscopy training characteristics, and including questions about the patterns of use and dermatologists' attitudes and opinions about dermoscopy. The questionnaire did not include any personal identification information and was translated in all the participating countries' languages. It was intended for all licensed dermatologists registered in European countries and was administered as an online survey in 32 participating countries. The dissemination of the survey in each country occurred through the national contact databases of dermatologists, under the responsibility of National Coordinating Teams, who collaborated with national dermatology and dermoscopy professional associations and were led by a National Coordinator elected from the members of the IDS Board of Directors or the Country Coordinators of Euromelanoma campaign. Online responses were collected through the IDS web- based tool for online surveys, into an access-restricted central database, grouped by country access code. Data cleaning of the study database was performed by three independent investigators (GG, AMF and PT).

The current work focuses on the answers to questions regarding the opinion of dermatologists on the impact of dermoscopy in increasing melanoma detection and reducing the number of unnecessary biopsies in their daily practice.

For statistical analysis, R software ${ }^{25}$ was used. Comparing proportions of two groups' chi-squared test and comparing proportions of ordered groups' chi-squared test for trends in proportions were used. Continuous data are given as means and standard deviations unless stated otherwise, and parametric tests for comparing groups were only used if corresponding assumptions were met. For multivariate analysis, all variables significant in univariate analysis were entered to a model with backwards elimination, controlled for sex, age, years in practice and numbers of (skin cancer and overall) patients per month. Remaining significant predictors are given as odds ratios (OR) with 95\% confidence intervals $(\mathrm{CI})$. A $P$-value $<0.05$ was regarded statistically significant. In univariate analyses $P$-values were adjusted by the method of Holm. ${ }^{26}$

\section{Results}

We collected 8519 responses from 32 countries in which a total number of 38300 dermatologists were registered as for the year 2014. After the data cleaning, 7480 valid responses were retained for analysis, of which 6602 reported to use dermoscopy. These were further analysed to assess the perceived impact of dermoscopy on the number of detected melanomas and on the number of unnecessary biopsies of benign lesions.

\section{Factors associated with a perceived benefit of} dermoscopy use in improving recognition of melanoma

Eighty-six per cent of all 6602 dermoscopy users reported that dermoscopy improved their ability to recognize melanoma compared with the naked-eye clinical examination (Table 1a). This positive perception showed a statistically significant association with younger age, working in public healthcare facilities, shorter duration of practising dermatology, higher number of patients seen/month, with receiving dermoscopy training during residency, and having trained in dermoscopy by any interactive form of education (courses, online courses, conferences, training with mentor/tutor) (Table 1a). It was also associated with the use of polarized light dermoscopy and digital dermoscopy, and with more frequent use of dermoscopy in their practice (Table 1b). Dermatologists who felt that dermoscopy improved their melanoma recognition were more likely to use the ABCD rule and pattern analysis than dermatologists who did not perceive a benefit of dermoscopy in increasing melanoma detection ( $31 \%$ vs. $26 \%$ and $31 \%$ vs. $25 \%$, respectively, $P=0.01$ ). Using no particular algorithm regularly was reported by $41 \%$ of 
Table 1 Factors associated with the perception that dermoscopy use improved melanoma recognition in the daily practice. (a) Demographic and practice setting factors associated with the perceived benefit of dermoscopy in the recognition of melanoma in the daily practice. (b) Practice factors associated with a perceived benefit of dermoscopy for the recognition of melanoma in the daily practice

(a)

N $=6228$
Female participants
Age (mean)
Place of work
Individual private practice
Private ambulatory hospital
Public ambulatory hospital
University hospital
Involved in teaching for dermatology residents
Years as dermatology specialist (mean)
No. of patients seen/month (mean)
No. of skin cancer patients seen/month (mean)
Dermoscopy training during residency
Types of dermoscopy training received outside residency
Dermoscopy course
Online dermoscopy course
Attended conferences/congresses
Books/atlases
Mentor/tutor
No training
I)

(b)

Duration of dermoscopy practice
$<2$ years
$2-5$ years
$>5$ years
Types of dermoscopes used

Types of dermoscopes used

Non-polarized immersion contact

Polarized light dermoscope

Dermoscope with digital camera

Digital videodermatoscopy system

Average frequency of using dermoscopy
$<1 \times$ /month
$1-4 /$ month
$>1 \times /$ week
Daily

Regularly used dermoscopic algorithm

ABCD rule

$\mathrm{CASH}$

Menzies algorithm

Seven-point checklist

Pattern analysis

No particular algorithm
Do you feel that using dermoscopy has increased the number of melanomas that you detected, in comparison with the naked-eye examination?

\begin{tabular}{|c|c|c|}
\hline Yes & No & $P$-value \\
\hline $5373(86.27 \%)$ & $855(13.73 \%)$ & \\
\hline $67.23 \%, n=3594$ & $67.37 \%, n=574$ & 1.000 \\
\hline 46.6 (SD: 10.93) & 49.37 (SD: 10.98) & $<0.001$ \\
\hline $36.57 \%, n=1965$ & $49.12 \%, n=420$ & $<0.001$ \\
\hline $19.75 \%, n=1061$ & $21.99 \%, n=188$ & 0.702 \\
\hline $31.92 \%, n=1715$ & $23.27 \%, n=199$ & $<0.001$ \\
\hline $21.11 \%, n=1134$ & $22.81 \%, n=195$ & 0.836 \\
\hline $12.99 \%, n=698$ & $12.28 \%, n=105$ & 1.000 \\
\hline 15.79 (SD: 10.63) & 18.53 (SD: 10.68) & $<0.001$ \\
\hline 442.84 (SD: 413.95$)$ & 395.71 (SD: 323.73) & 0.002 \\
\hline 61.02 (SD:102.64) & 51.69 (SD:140.91) & 0.413 \\
\hline $44.72 \%, n=2374$ & $24.11 \%, n=203$ & $<0.001$ \\
\hline $66.65 \%, n=3581$ & $45.61 \%, n=390$ & $<0.001$ \\
\hline $19.17 \%, n=1030$ & $13.45 \%, n=115$ & 0.001 \\
\hline $73.14 \%, n=3930$ & $66.78 \%, n=571$ & 0.002 \\
\hline $81.00 \%, n=4352$ & $77.43 \%, n=662$ & 0.163 \\
\hline $24.49 \%, n=1316$ & $16.73 \%, n=143$ & $<0.001$ \\
\hline $3.03 \%, n=163$ & $4.33 \%, n=37$ & 0.413 \\
\hline
\end{tabular}

Do you feel that using dermoscopy has increased the number of melanomas that you detected, in comparison with the naked-eye examination?

\begin{tabular}{|c|c|c|}
\hline Yes & No & $P$-value \\
\hline $10.74 \%, n=575$ & $11.87 \%, n=101$ & 0.256 \\
\hline $19.56 \%, n=1047$ & $22.91 \%, n=195$ & \\
\hline $69.70 \%, n=3731$ & $65.22 \%, n=555$ & \\
\hline $53.17 \%, n=2857$ & $57.08 \%, n=488$ & 0.294 \\
\hline $53.97 \%, n=2900$ & $47.72 \%, n=408$ & 0.008 \\
\hline $24.01 \%, n=1290$ & $14.62 \%, n=125$ & $<0.001$ \\
\hline $27.55 \%, n=1480$ & $15.56 \%, n=133$ & $<0.001$ \\
\hline $0.84 \%, n=45$ & $1.29 \%, n=11$ & $<0.001$ \\
\hline $3.86 \%, n=207$ & $6.44 \%, n=55$ & \\
\hline $10.39 \%, n=557$ & $13.47 \%, n=115$ & \\
\hline $84.91 \%, n=4553$ & $78.81 \%, n=673$ & \\
\hline $31.23 \%, n=1678$ & $25.50 \%, n=218$ & 0.011 \\
\hline $0.69 \%, n=37$ & $0.35 \%, n=3$ & 1.000 \\
\hline $2.62 \%, n=141$ & $2.57 \%, n=22$ & 1.000 \\
\hline $8.32 \%, n=447$ & $5.73 \%, n=49$ & 0.137 \\
\hline $31.01 \%, n=1666$ & $24.91 \%, n=213$ & 0.005 \\
\hline $27.40 \%, n=1472$ & $41.05 \%, n=351$ & $<0.001$ \\
\hline
\end{tabular}

$P$ values $<0.05$ are highlighted in bold. 
dermatologists who did not feel that dermoscopy improved their melanoma detection ability, compared to $27 \%$ of those who considered dermoscopy to be useful for increased melanoma detection $(P<0.001)$ (Table $1 \mathrm{~b})$. Dermatologists who reported a benefit of dermoscopy in increasing their melanoma detection reported higher self-confidence in their dermoscopic diagnostic skills for all categories of inflammatory and neoplastic skin diseases and were more likely to have positive opinions about dermoscopy's utility and benefits for the practice (Table S1, Supporting Information), in comparison with the dermatologists who did not consider that dermoscopy use increased the melanoma detection in their practice.

In multivariate analysis, the following factors remained significantly associated with perceived improvement of melanoma recognition (Table 2): working in a public healthcare facility, dermoscopy training during residency, receiving any kind of dermoscopy training except atlases/books, positive opinion about the utility of dermoscopy in monitoring non-melanocytic lesions and self-confidence in the assessment of pigmented lesions. Dermatologists who did not use any particular algorithm were less likely to perceive a benefit of dermoscopy in increasing melanoma detection (OR 0.814, 95\% CI: 0.675-0.985).

\section{Factors associated with the perceived benefit of dermoscopy in reducing the number of unnecessary biopsies}

The majority $(70.7 \%)$ of dermoscopy users observed that dermoscopy allowed them to reduce the number of unnecessary biopsies of benign lesions (Table 3a). This positive perception was associated with the following: working in private practice or in university hospitals, shorter duration of practising dermatology, higher number of patients and skin cancer patients seen per month, having received dermoscopy training during residency and having received dermoscopy training in the form of courses, conferences or atlases/books (Table 3a). It was associated also with longer dermoscopy practice, with the use of polarized light dermoscopy and of digital dermoscopy, with more frequent use of this technique and with using pattern analysis for the dermoscopic diagnosis (Table $3 \mathrm{~b}$ ). Use of the ABCD rule or using no particular algorithm at all was reported more frequently by dermatologists who have not perceived any benefit of dermoscopy in reducing biopsies (Table 3b). Further, self-confidence in the dermoscopic skills for all categories of inflammatory and tumoral skin diseases, and positive opinions about dermoscopy's advantages for the practice were associated with a perceived benefit of dermoscopy in decreasing the number of unnecessary excisions (Table S2, Supporting Information).

In multivariate analysis, the following factors remained significantly associated with perceived reduction in the number of unnecessary biopsies (Table 4): working in private practice, shorter duration of practising dermatology, training in dermoscopy in the form of attending conferences, longer duration of practising dermoscopy and more frequent use of this technique, use of digital dermoscopy devices and self-confidence in the dermoscopic diagnostic skills for pigmented lesions. Using the $\mathrm{ABCD}$ rule or no particular algorithm decreased the

Table 2 Factors associated with a perceived benefit of dermoscopy for the improvement of melanoma recognition in the daily practice (multivariate analysis)

\begin{tabular}{|c|c|c|c|c|}
\hline Dermoscopy use increased detected melanoma & OR & $2.5 \% \mathrm{Cl}$ & $97.5 \% \mathrm{Cl}$ & $P$-value \\
\hline \multicolumn{5}{|l|}{ Place of work } \\
\hline Individual private practice & 0.791 & 0.640 & 0.977 & 0.029 \\
\hline Private ambulatory/hospital & 0.736 & 0.585 & 0.929 & 0.009 \\
\hline Public ambulatory/hospital & 1.573 & 1.269 & 1.959 & $<0.001$ \\
\hline Dermoscopy training during residency & 1.485 & 1.198 & 1.847 & $<0.001$ \\
\hline \multicolumn{5}{|l|}{ Dermoscopy training } \\
\hline Dermoscopy course & 1.611 & 1.336 & 1.942 & $<0.001$ \\
\hline Online dermoscopy course & 1.362 & 1.057 & 1.773 & 0.019 \\
\hline Attended Congresses & 1.293 & 1.056 & 1.580 & 0.012 \\
\hline Mentor/Tutor & 1.353 & 1.073 & 1.717 & 0.012 \\
\hline \multicolumn{5}{|l|}{ Regularly used dermoscopic algorithm } \\
\hline No used algorithm & 0.814 & 0.675 & 0.985 & 0.033 \\
\hline \multicolumn{5}{|l|}{ Opinion on the utility of dermoscopy in the following situations } \\
\hline Follow-up of non-melanocytic skin lesions ${ }^{*}$ & 1.659 & 1.347 & 2.046 & $<0.001$ \\
\hline \multicolumn{5}{|l|}{ Self-confidence in the dermoscopic diagnosis of } \\
\hline Pigmented skin tumours ${ }^{\star}$ & 1.626 & 1.175 & 2.232 & 0.003 \\
\hline \multicolumn{5}{|l|}{ Perceived advantages of dermoscopy use } \\
\hline Increases confidence of clinical diagnosis* & 2.361 & 1.141 & 5.015 & 0.022 \\
\hline Reduces the number of unnecessary biopsies/excisions * & 1.769 & 1.311 & 2.369 & $<0.001$ \\
\hline
\end{tabular}

OR, odds Ratio; $\mathrm{Cl}$, confidence interval.

*Variables with a calculated linear correlation. 
Table 3 Factors associated with perceived benefit o dermoscopy in reducing unnecessary benign biopsies. (a) Demographic factors associated with the perceived benefit of dermoscopy in reducing the number of unnecessary biopsies of benign lesions. (b) Practice factors associated with perceived benefit of dermoscopy in reducing unnecessary biopsies of benign lesions

(a)

Female participants

Age (mean)

Place of work

Individual private practice

Private ambulatory/hospital

Public ambulatory/hospital

University hospital

Years as dermatology specialist (mean)

Patients seen/month (mean)

Skin cancer patients seen/month (mean)

Dermoscopy training during residency

Types of dermoscopy training received outside residency

Dermoscopy course

Online dermoscopy course

Attended congresses

Books atlases

Mentor tutor

No training

(b)
Involved in teaching activity for dermatology residents

In your practice, how did the use of dermoscopy influence the number of excisions of benign lesions that you performed?

\begin{tabular}{|c|c|c|}
\hline $\begin{array}{l}\text { Reduced excisions } \\
\text { of benign lesions }\end{array}$ & $\begin{array}{l}\text { Did not reduce excisions } \\
\text { of benign lesions }\end{array}$ & $P$-value \\
\hline 4406 (70.76\%) & $1820(29.24 \%)$ & NA \\
\hline $66.36 \%, n=2906$ & $69.33 \%, n=1259$ & 0.178 \\
\hline 47.09 (SD: 10.81) & 46.74 (SD: 11.4) & 0.884 \\
\hline $40.06 \%, n=1765$ & $34.45 \%, n=627$ & $<0.001$ \\
\hline $18.02 \%, n=794$ & $25.11 \%, n=457$ & $<0.001$ \\
\hline $29.05 \%, n=1280$ & $34.56 \%, n=629$ & $<0.001$ \\
\hline $22.47 \%, n=990$ & $18.35 \%, n=334$ & 0.004 \\
\hline $13.12 \%, n=578$ & $12.31 \%, n=224$ & 0.884 \\
\hline 15.94 (SD: 10.54) & 16.79 (SD: 11.01) & 0.044 \\
\hline 459.02 (SD: 418.65) & 384.17 (SD:357.31) & $<0.001$ \\
\hline 66.47 (SD: 114.12) & 43.21 (SD: 92.11) & $<0.001$ \\
\hline $44.94 \%, n=1954$ & $34.33 \%, n=618$ & $<0.001$ \\
\hline $67.48 \%, n=2973$ & $54.73 \%, n=996$ & $<0.001$ \\
\hline $18.86 \%, n=831$ & $17.36 \%, n=316$ & 0.884 \\
\hline $75.44 \%, n=3324$ & $64.89 \%, n=1181$ & $<0.001$ \\
\hline $81.59 \%, n=3595$ & $77.86 \%, n=1417$ & 0.008 \\
\hline $23.88 \%, n=1052$ & $22.25 \%, n=405$ & 0.884 \\
\hline $2.59 \%, n=114$ & $4.73 \%, n=86$ & $<0.001$ \\
\hline
\end{tabular}

In your practice, how did the use of dermoscopy influence the number of excisions of benign lesions that you performed?

\begin{tabular}{|c|c|c|}
\hline $\begin{array}{l}\text { Reduced excisions of } \\
\text { benign lesions }\end{array}$ & $\begin{array}{l}\text { Did not reduce excisions } \\
\text { of benign lesions }\end{array}$ & $\boldsymbol{P}$-value \\
\hline $7.25 \%, n=318$ & $19.67 \%, n=357$ & $<0.001$ \\
\hline $17.98 \%, n=789$ & $24.85 \%, n=451$ & \\
\hline $74.77 \%, n=3280$ & $55.48 \%, n=1007$ & \\
\hline $52.81 \%, n=2327$ & $55.88 \%, n=1017$ & 0.178 \\
\hline $56.20 \%, n=2476$ & $45.66 \%, n=831$ & $<0.001$ \\
\hline $24.26 \%, n=1069$ & $18.90 \%, n=344$ & $<0.001$ \\
\hline $29.23 \%, n=1288$ & $17.75 \%, n=323$ & $<0.001$ \\
\hline $0.48 \%, n=21$ & $1.82 \%, n=33$ & $<0.001$ \\
\hline $2.39 \%, n=105$ & $8.64 \%, n=157$ & \\
\hline $8.51 \%, n=374$ & $16.45 \%, n=299$ & \\
\hline $88.63 \%, n=3896$ & $73.10 \%, n=1329$ & \\
\hline $27.71 \%, n=1221$ & $36.98 \%, n=673$ & $<0.001$ \\
\hline $0.61 \%, n=27$ & $0.71 \%, n=13$ & 0.884 \\
\hline $2.88 \%, n=127$ & $1.92 \%, n=35$ & 0.266 \\
\hline $8.37 \%, n=369$ & $6.87 \%, n=125$ & 0.308 \\
\hline $33.66 \%, n=1483$ & $21.92 \%, n=399$ & $<0.001$ \\
\hline $27.78 \%, n=1224$ & $32.86 \%, n=598$ & 0.001 \\
\hline
\end{tabular}

Seven-point checklist
Duration of dermoscopy practice

$<2$ years
$2-5$ years
$>5$ years

Types of dermoscopes used

Non-polarized immersion contact

Polarized light dermoscope

Dermoscope with digital camera

Digital videodermatoscopy system

Average frequency of using dermoscopy
$<1 \times /$ month
1-4/month
$>1 \times /$ week
Daily

Regularly used dermoscopic algorithm

$A B C D$ rule

$\mathrm{CASH}$

Menzies algorithm

Pattern analysis

No particular algorithm

-

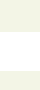

(n)

27

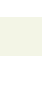

5

$P$ values $<0.05$ are highlighted in bold. 
Table 4 Factors associated with a perceived benefit in reducing excisions of benign lesions (multivariate analysis)

\begin{tabular}{|c|c|c|c|c|}
\hline Dermoscopy-reduced excisions of benign lesions & OR & $2.5 \% \mathrm{Cl}$ & $97.5 \% \mathrm{Cl}$ & $P$-value \\
\hline Age & 1.029 & 1.013 & 1.045 & $<0.001$ \\
\hline Number of years of dermatology practice & 0.963 & 0.948 & 0.978 & $<0.001$ \\
\hline \multicolumn{5}{|l|}{ Place of work } \\
\hline Individual private practice & 1.211 & 1.031 & 1.423 & 0.020 \\
\hline Private ambulatory hospital & 0.836 & 0.704 & 0.994 & 0.042 \\
\hline \multicolumn{5}{|l|}{ Type of dermoscopy training received outside residency } \\
\hline Attended congresses & 1.170 & 1.001 & 1.365 & 0.047 \\
\hline Duration of dermoscopy practice ${ }^{*}$ & 1.552 & 1.300 & 1.852 & $<0.001$ \\
\hline \multicolumn{5}{|l|}{ Types of dermoscopes used } \\
\hline Dermoscope with digital camera & 1.188 & 1.006 & 1.406 & 0.044 \\
\hline Digital videodermatoscopy system & 1.274 & 1.073 & 1.515 & 0.006 \\
\hline High frequency of using dermoscopy* & 1.691 & 1.068 & 2.709 & 0.026 \\
\hline \multicolumn{5}{|l|}{ Particular dermoscopic algorithm regularly used } \\
\hline$A B C D$ rule & 0.666 & 0.564 & 0.786 & $<0.001$ \\
\hline No used algorithm & 0.707 & 0.598 & 0.835 & $<0.001$ \\
\hline \multicolumn{5}{|c|}{ Self-confidence in the dermoscopic assessment of the following } \\
\hline Pigmented skin tumours* & 1.430 & 1.078 & 1.899 & 0.013 \\
\hline Inflammatory skin lesions ${ }^{\star}$ & 0.707 & 0.611 & 0.820 & $<0.001$ \\
\hline \multicolumn{5}{|l|}{ Perceived advantages of dermoscopy use } \\
\hline Reduces the number of unnecessary biopsies/excisions ${ }^{*}$ & 5.396 & 4.023 & 7.384 & $<0.001$ \\
\hline
\end{tabular}

OR, odds ratio; $\mathrm{Cl}$, confidence interval.

*Variables with a calculated linear correlation.

likelihood to report a benefit of dermoscopy in reducing unnecessary excisions (OR 0.66, 95\% CI: $0.56-0.78$ and OR 0.70, 95\% CI: $0.59-0.83$, respectively).

\section{Discussion}

The ultimate goal of dermoscopy for melanocytic lesions is to increase the early recognition of melanoma while reducing the number of invasive procedures needed for diagnosis. The capacity of dermoscopy to achieve this goal, increasing the sensitivity and specificity of melanoma diagnosis, has been solidly documented in multiple studies. ${ }^{9,10,27}$ Hence, it is natural to hypothesize that making more widespread and better use of this accessible and affordable technique could have an impact at population-based scale, on improving melanoma prognosis through earlier detection, while reducing diagnostic costs. Testing this hypothesis is challenging and first requires understanding the current place and impact of dermoscopy in the real-life dermatology practice, as well as the drivers and barriers for future improvement. Our study performed the largest survey of dermatologists so far, as a step towards this understanding.

Our results confirm at pan-European scale that dermoscopy is a useful tool for the practice of dermatologists, allowing them to detect more melanomas and to reduce the number of unnecessary benign excisions performed. Our findings reinforce the crucial importance of proper training in translating the potential advantages of dermoscopy into real benefits for melanoma diagnosis. Notably, dermoscopy training during residency almost doubled the proportion of dermatologists reporting that dermoscopy allowed them to detect more melanomas than naked-eye examination alone (Table 1a), and this effect was maintained in the multivariate analysis (OR 1.51, 95\% CI: $1.22-$ 1.88) (Table 2). Residency dermoscopy training also related, although less markedly, to the reported benefit of dermoscopy in reducing the number of unnecessary biopsies.

To our knowledge, this is the first study that explores the impact of dermoscopy training during dermatology residency on the subsequent dermatology practice across Europe. Dermoscopy training during residency has been shown to improve the opinions about dermoscopy, increase the self-confidence in the skin cancer diagnosis and improve the diagnostic accuracy of dermatology residents in USA. ${ }^{28-31}$ Such studies were lacking in Europe. Dermatology residency curricula are diverse across the European continent, not all include dermoscopy training or have included it only recently, and the actual form of training is also highly heterogeneous. Given that dermoscopy training is relatively new, it is notable that $38 \%$ of all European dermatologists participating in our study reported dermoscopy training during residency.

In our study, dermatologists who trained in dermoscopy through interactive methods (courses, conferences, mentoring/ tutoring) were more likely (OR 1.36-1.64, Table 2) to report increased melanoma recognition through dermoscopy, while this effect was not seen for training through atlases/books. This trend was less clear in regard to the reduction in unnecessary biopsies, and further research on the most efficient forms of dermoscopy training for melanoma diagnosis is warranted. 
The greater experience with dermoscopy for skin cancer detection, as reflected through more years of dermoscopy practice and higher number of patients with skin cancer seen per month, appeared relevant for the reduction in unnecessary biopsies, but less so for increased melanoma recognition. This concurs with previous reports showing that even brief dermoscopy training in inexperienced users can improve the sensitivity of melanoma recognition, ${ }^{9,32-34}$ but that the increase in specificity occurred mostly at an expert level and in specialized centres. ${ }^{18,19,21}$

A positive impact of dermoscopy on melanoma detection was expectedly associated with more frequent use of dermoscopy, across all disease categories, as well as with self-confidence in dermoscopic skills for pigmented lesions. Expertise, experience and confidence seem to engage in a positive feedback loop that enhances the use and the benefits of dermoscopy. This supports the argument that investment in training and in providing dermatologists with the opportunity to use dermoscopy could also improve the efficient use of this technique, for better detection at lower costs. Consistent with our findings, a recent study ${ }^{35}$ used a melanoma disease model to demonstrate that adequate dermoscopy training of dermatologists is cost-effective, in terms of increasing patients' quality-adjusted life-years (QALY) and lowering the medical costs. In this context, it is noteworthy that only $56 \%$ of dermoscopy users reported confidence in their skills for the diagnosis of pigmented lesions, although this is the main indication and topic of training in dermoscopy. Optimizing educational efforts are needed, and the question remains open how to achieve this. A more detailed analysis of our study data regarding the patterns of dermoscopy training across Europe, and their consequence for the dermatologists' practice, is ongoing and will be reported in the future.

In our study, the use of polarized light dermoscopy and digital dermoscopy was associated with a perceived benefit of dermoscopy both for improving melanoma recognition and for reducing unnecessary excisions. Monitoring melanocytic lesions by means of sequential digital dermoscopy has been demonstrated to increase the early detection of thinner melanoma and to lower the NNE rates, increasing dermoscopy's cost benefits. ${ }^{12,36-41}$ This approach is increasingly recommended by the current European guidelines of melanoma management. ${ }^{14,16,17}$ Our results confirm the importance of access to and use of digital dermoscopy for increasing the performance of melanoma diagnosis in dermatologist practice Europe-wide. As $35.7 \%$ of our responding dermatologists reported the use of a form of digital dermoscopy, this leaves room for significant improvement in the future, while more research is needed to establish the true cost-effectiveness of widespread use of digital dermoscopy for melanoma early detection.

The classic ABCD rule and pattern analysis were the most frequently reported by dermatologists, likely because they are the oldest and the most widely taught algorithms. They also represent two diverging concepts of diagnostic approach, heuristic and analytical, both subjects of a long-term debate over which is superior. ${ }^{40,41}$ It was noteworthy that the use of the ABCD rule was associated with increased melanoma recognition (Table $1 \mathrm{~b}$ ) but decreased the likelihood for reducing the number of unnecessary biopsies (Table 4); pattern analysis increased both melanoma recognition (sensitivity) and the specificity of diagnosis (Tables $1 \mathrm{~b}$ and $3 \mathrm{~b}$ ). These findings suggest that pattern analysis is the best algorithm for increasing accuracy of melanoma diagnosis and should be emphasized in the training of dermoscopy. Nonetheless, using any algorithm vs. no algorithm at all was better for increasing melanoma recognition and reducing the number of unnecessary biopsies.

The impact of dermoscopy on the diagnostic performance for melanoma varied according to the practice setting for European dermatologists, with two particular contrasting scenarios standing out. On the one hand, dermatologists working in individual private practices were less likely to report a benefit of dermoscopy to increase detection of melanomas, but noted greater benefit in reducing the number of unnecessary biopsies. Conversely, dermatologists working in public healthcare facilities experience the most benefit of dermoscopy in increasing melanoma detection, but were less likely to report a reduction in the number of biopsies. For individual practices, it might be argued that they face a greater pressure to reduce costs and to limit invasive procedures for their patients, so the main benefit is perceived in this area. Dermoscopy appears to fulfil the role of screening for melanoma, which is an important task of public healthcare facilities, attending for the vast majority of patients in the European health systems. However, screening appears to occur without significant reduction in unnecessary biopsies, and hence of the costs for public healthcare - not a good omen in the current landscape of austerity in healthcare budgets. It is thus of vital importance to examine how to further reduce biopsies by dermoscopy also in public hospitals. Our prior finding ${ }^{24}$ had revealed that dermatologists working in public facilities were the least likely to use dermoscopy at all, or if they used it, it was less intensively; they were also the most likely to report the lack of dermoscopy equipment as a barrier to use dermoscopy. Therefore, proper training and improved access to dermoscopy equipment, especially digital, may be the main ways to address this issue in public hospitals.

Our analysis has several limitations. The main one is the subjective nature of the responses. The difference in melanoma diagnosis numbers or in NNEs through dermoscopy use could not be verified from the practice records of such a large pan-European sample of respondents. Similarly, our study allowed for a qualitative, but not quantitative evaluation of the benefit of dermoscopy for melanoma detection and reducing the unnecessary biopsies. Nonetheless, our results are consistent with previous national surveys ${ }^{23,42}$ and with the evidence of the effect of dermoscopy on diagnostic accuracy while providing an unprecedented insight into dermatologists' perceptions on the role of dermoscopy. We acknowledge that responses are likely influenced by the background factors related to the national systems 
of dermatology training and healthcare practice, which are highly heterogeneous in Europe. However, the free movement of people, including widespread mobility of the patients, physicians and medical trainees, is a fundamental concept in Europe, and medical licenses are virtually automatically recognized in EU countries. Therefore, a common European vision is needed and all efforts must be made to ensure that disparities between countries $^{43}$ are narrowed and the quality of medical training and care becomes de facto similar throughout the continent. In this perspective, our pan-European results are particularly informative.

\section{Conclusion}

Our pan-European survey confirms that dermoscopy is a valuable tool to improve melanoma recognition and reduce the number of invasive diagnostic procedures in the daily dermatology practice across the continent. However, in many cases, this technique is not used to its full potential. The study brings compelling evidence that enhancing dermoscopy training, especially during dermatology residency, and increasing dermatologists' access to dermoscopy equipment, especially digital dermoscopy, would contribute significantly to improving the accuracy of melanoma diagnosis in Europe, with potential to alleviate the current disparities in early detection and prognosis of this deadly tumour.

\section{Acknowledgements}

Special thanks to Gerald Gabler, IDS webmaster, who accomplished the essential tasks of creating the study web page, of setting up and maintaining the online survey for 32 participating countries, of creating the central online study database and for participating in the data cleaning. Thanks to all the members of National Coordinating Teams for their efforts in translating the questionnaires, disseminating the survey, motivating the dermatologist colleagues to respond and collecting offline answers. Their names are listed on the Eurodermoscopy website.

\section{References}

1 Errichetti E, Stinco G. The practical usefulness of dermoscopy in general dermatology. G Ital Dermatol Venereol 2015; 150: 533-546.

2 Giacomel J, Zalaudek I, Argenziano G et al. Dermoscopy of hypertrophic lupus erythematosus and differentiation from squamous cell carcinoma. $J$ Am Acad Dermatol 2015; 72: S33-S36.

3 Goncharova Y, Attia EA, Souid K et al. Dermoscopic features of clinically inflammatory dermatoses and their correlation with histopathologic reaction patterns. Arch Dermatol Res 2015; 307: 23-30.

4 Haliasos EC, Kerner M, Jaimes-Lopez $\mathrm{N}$ et al. Dermoscopy for the pediatric dermatologist part I: dermoscopy of pediatric infectious and inflammatory skin lesions and hair disorders. Pediatr Dermatol 2013; 30: 163-171.

5 Tosti A, Torres F. Dermoscopy in the diagnosis of hair and scalp disorders. Actas Dermosifiliogr 2009; 100(Suppl 1): 114-119.

6 Tschandl P, Argenziano G, Bakos R et al. Dermoscopy and entomology (entomodermoscopy). J Dtsch Dermatol Ges 2009; 7: 589-596.

7 Zalaudek I, Argenziano G. Dermoscopy of actinic keratosis, intraepidermal carcinoma and squamous cell carcinoma. Curr Probl Dermatol 2015; 46: 70-76.

8 Zalaudek I, Kreusch J, Giacomel J et al. How to diagnose nonpigmented skin tumors: a review of vascular structures seen with dermoscopy: part
II. Nonmelanocytic skin tumors. J Am Acad Dermatol 2010; 63: 377-386; quiz 87-8.

9 Kittler H, Pehamberger H, Wolff K et al. Diagnostic accuracy of dermoscopy. Lancet Oncol 2002; 3: 159-165.

10 Vestergaard M, Macaskill P, Holt P et al. Dermoscopy compared with naked eye examination for the diagnosis of primary melanoma: a metaanalysis of studies performed in a clinical setting. Br J Dermatol 2008; 159: 669-676.

11 Rosendahl C, Williams G, Eley D et al. The impact of subspecialization and dermatoscopy use on accuracy of melanoma diagnosis among primary care doctors in Australia. J Am Acad Dermatol 2012; 67: 846-852.

12 Salerni G, Teran T, Puig S et al. Meta-analysis of digital dermoscopy follow-up of melanocytic skin lesions: a study on behalf of the International Dermoscopy Society. J Eur Acad Dermatol Venereol 2013; 27: 805-814.

13 Argenziano G, Albertini G, Castagnetti F et al. Early diagnosis of melanoma: what is the impact of dermoscopy? Dermatol Ther 2012; 25: 403 409.

14 Garbe C, Peris K, Hauschild A et al. Diagnosis and treatment of melanoma. European consensus-based interdisciplinary guideline-Update 2012. Eur J Cancer 2012; 48: 2375-2390.

15 Marsden JR, Newton-Bishop JA, Burrows L et al. Revised U.K. guidelines for the management of cutaneous melanoma 2010. Br J Dermatol 2010; 163: 238-256.

16 Pflugfelder A, Kochs C, Blum A et al. Malignant melanoma S3-guideline "diagnosis, therapy and follow-up of melanoma". J Dtsch Dermatol Ges 2013; 11(Suppl 6): 1-116, 1-26.

17 Watts CG, Dieng M, Morton RL et al. Clinical practice guidelines for identification, screening and follow-up of individuals at high risk of primary cutaneous melanoma: a systematic review. Br J Dermatol 2015; 172: 33-47.

18 van der Rhee JI, Bergman W, Kukutsch NA. The impact of dermoscopy on the management of pigmented lesions in everyday clinical practice of general dermatologists: a prospective study. Br J Dermatol 2010; 162: 563 567.

19 van der Rhee JI, Bergman W, Kukutsch NA. Impact of dermoscopy on the management of high-risk patients from melanoma families: a prospective study. Acta Derm Venereol 2011; 91: 428-431.

20 Carli P, De Giorgi V, Crocetti E et al. Improvement of malignant/benign ratio in excised melanocytic lesions in the 'dermoscopy era': a retrospective study 1997-2001. Br J Dermatol 2004; 150: 687-692.

21 Argenziano G, Cerroni L, Zalaudek I et al. Accuracy in melanoma detection: a 10-year multicenter survey. J Am Acad Dermatol 2012; 67: 54-59.

22 Argenziano G, Moscarella E, Annetta A et al. Melanoma detection in Italian pigmented lesion clinics. G Ital Dermatol Venereol 2014; 149: 161166.

23 Moulin C, Poulalhon N, Duru G et al. Dermoscopy use by French private practice dermatologists: a nationwide survey. Br J Dermatol 2013; 168: 74-79.

24 Forsea AM, Tschandl P, Del Marmol V et al. Factors driving the use of dermoscopy in Europe: a Pan-European survey. Br J Dermatol 2016; 175: 1329-1337.

25 Team RC. R: A Language and Environment for Statistical Computing. R Foundation for Statistical Computing, Vienna, Austria, 2015. URL https://www.R-project.org/.

26 Holm S. A simple sequentially rejective multiple test procedure. Scand J Stat 1979; 6: 65-70.

27 Menzies SW. Evidence-based dermoscopy. Dermatol Clin 2013; 31: 521524, vii.

$28 \mathrm{Wu}$ TP, Newlove T, Smith L et al. The importance of dedicated dermoscopy training during residency: a survey of US dermatology chief residents. J Am Acad Dermatol 2013; 68: 1000-1005.

29 Nehal KS, Oliveria SA, Marghoob AA et al. Use of and beliefs about dermoscopy in the management of patients with pigmented lesions: a survey of dermatology residency programmes in the United States. Melanoma Res 2002; 12: 601-605. 
30 Terushkin V, Oliveria SA, Marghoob AA et al. Use of and beliefs about total body photography and dermatoscopy among US dermatology training programs: an update. J Am Acad Dermatol 2010; 62: 794-803.

31 Engasser HC, Warshaw EM. Dermatoscopy use by US dermatologists: a cross-sectional survey. J Am Acad Dermatol 2010; 63: 412-419, 9.e1-2.

32 Chevolet I, Hoorens I, Janssens A et al. A short dermoscopy training increases diagnostic performance in both inexperienced and experienced dermatologists. Australas J Dermatol 2015; 56: 52-55.

33 Argenziano G, Puig S, Zalaudek I et al. Dermoscopy improves accuracy of primary care physicians to triage lesions suggestive of skin cancer. $J$ Clin Oncol 2006; 24: 1877-1882.

34 Koelink CJ, Vermeulen KM, Kollen BJ et al. Diagnostic accuracy and cost-effectiveness of dermoscopy in primary care: a cluster randomized clinical trial. J Eur Acad Dermatol Venereol 2014; 28: 1442-1449.

35 Tromme I, Legrand C, Devleesschauwer B et al. Cost-effectiveness analysis in melanoma detection: a transition model applied to dermoscopy. Eur J Cancer 2016; 67: 38-45.

36 Haenssle HA, Krueger U, Vente C et al. Results from an observational trial: digital epiluminescence microscopy follow-up of atypical nevi increases the sensitivity and the chance of success of conventional dermoscopy in detecting melanoma. J Invest Dermatol 2006; 126: 980-985.

37 Menzies SW, Emery J, Staples M et al. Impact of dermoscopy and shortterm sequential digital dermoscopy imaging for the management of pigmented lesions in primary care: a sequential intervention trial. Br J Dermatol 2009; 161: 1270-1277.

38 Burroni M, Wollina U, Torricelli R et al. Impact of digital dermoscopy analysis on the decision to follow up or to excise a pigmented skin lesion: a multicentre study. Skin Res Technol 2011; 17: 451-460.

39 Tromme I, Sacre L, Hammouch F et al. Availability of digital dermoscopy in daily practice dramatically reduces the number of excised melanocytic lesions: results from an observational study. Br J Dermatol 2012; 167: 778-786.

40 Salerni G, Carrera C, Lovatto L et al. Benefits of total body photography and digital dermatoscopy ("two-step method of digital follow-up") in the early diagnosis of melanoma in patients at high risk for melanoma. J Am Acad Dermatol 2012; 67: e17-e27.

41 Tromme I, Devleesschauwer B, Beutels P et al. Selective use of sequential digital dermoscopy imaging allows a cost reduction in the melanoma detection process: a belgian study of patients with a single or a small number of atypical nevi. PLoS One 2014; 9: e109339.

42 Butler TD, Matin RN, Affleck AG et al. Trends in dermoscopy use in the UK: results from surveys in 2003 and 2012. Dermatol Pract Concept 2015; 5: 29-38.

43 Forsea AM, Del Marmol V, Stratigos A et al. Melanoma prognosis in Europe: far from equal. Br J Dermatol 2014; 171: 179-182.

\section{Appendix 1}

\section{Eurodermoscopy Working Group Members}

M. Arenbergerova: Department of Dermatology, Third Medical Faculty, Charles University Prague, Czech Republic; A. Azenha: Hospital Privado da Trofa, Portugal; A. Blum: DermPrevOncol, Public, Private and Teaching Practice of Dermatology, Konstanz, Germany; J.C. Bowling: Private Practice Nuffield Hospital, Oxford, UK; R.P. Braun: Department of Dermatology, University Hospital of Zürich, Switzerland; M. Bylaite-Bucinskiene: Centre of Dermatovenereology, Vilnius University, Vilnius, Lithuania; L. Čabrijan: Department of Dermatovenereology, Clinical Hospital Center Rijeka, Rijeka, Croatia; H. Dobrev: Department of Dermatology and Venereology, Medical Faculty, Medical University, Plovdiv, Bulgaria; F. Özdemir: Dermato-
Oncology Unit, Department of Dermatology, Ege University, Medical Faculty, Izmir, Turkey; J. Hegyi: Institute of Clinical and Experimental Dermatovenereology, Bratislava, Slovak Republic; H. Helppikangas: Dermatology Department, Clinical Center, University of Sarajevo, Bosnia \& Herzegovina; R. Hofmann-Wellenhof: Department of Dermatology, Medical University Graz, Graz, Austria; R. Karls: Department of Infectology and dermatology, Riga Stradins University, Derma Clinic Riga, Latvia; U. Krumkachou: Dermatovenereology and Cosmetology Department, Belarusian Medical Academy of Post-Graduate Education, Minsk, Belarus; N. Kukutsch: Department of Dermatology, Leiden University Medical Center, The Netherlands; I. McCormack: Belfast Health \& Social Care Trust, Belfast, Ireland; L. Mekokishvili: Dermatovenereology Department at Petre Shotadze Tbilisi Medical Academy, Tbilisi, Georgia; N. Nathansohn: Department of Dermatology and the Advanced Technologies Center, C. Sheba Medical Center, Tel Hashomer, Israel; K. Nielsen: Lund University, Helsingborg Hospital, Department of Clinical Sciences Lund, Dermatology and Venereology, Lund, Sweden; J. Olah: Department of Dermatology and Allergology, University of Szeged, Szeged, Hungary; S. Puig: Melanoma Unit, Dermatology Department, Hospital Clínic \& IDIBAPS (Institut d'Investigacions Biomèdiques August Pi i Sunyer), Barcelona, Spain; Centro Investigación Biomédica en Red de Enfermedades Raras (CIBERER), Instituto de Salud Carlos III (ISCIII), Barcelona, Spain; Departament de Medicina, Universitat de Barcelona, Barcelona, Spain; P. Rubegni: Department of Medical and Surgical Science and Neuroscience, University of Siena, Siena, Italy; T. Planinsek Rucigaj: Dermatovenereological Clinic, University Medical Centre Ljubljana, Slovenia; T.R. Schopf: Norwegian Centre for E-health Research, University Hospital of North-Norway, Tromsø, Norway; V. Sergeev: Central Research Dermatology Clinic, Moscow; A. Stratigos: 1st Department of Dermatology - Venereology, National and Kapodistrian University of Athens School of Medicine, Andreas Sygros Hospital, Athens, Greece; L. Thomas: 1. Lyon 1 University; 2. Dermatology Center Hospitalier Lyon Sud; 3. Lyons Cancer Research Center INSERM U1052 - CNRS UMR5286 - Lyon France; D. Tiodorovic: Clinic of Dermatovenereology, Clinical Center of Nis, Medical Faculty, Nis, Serbia; A. Vahlberg: Vahlberg \& Pild Ltd, Tallinn, Estonia; Z. Zafirovik: University Clinic of Dermatology, Medical Faculty, University "St. Cyril and Methodius", Skopje, The Former Yugoslav Republic of Macedonia.

\section{Supporting information}

Additional Supporting Information may be found in the online version of this article:

Table S1. Attitudes associated with a perceived benefit of dermoscopy for the recognition of melanoma in the daily practice.

Table S2. Attitudes associated with a perceived benefit of dermoscopy in reducing unnecessary biopsies of benign lesions. 